\title{
ON A PROBLEM OF G. GRÄTZER
}

\author{
TIBOR KATRIŇÁK
}

\begin{abstract}
A characterization of the congruence lattice of a modular $p$ algebra in terms of congruence pairs is given.
\end{abstract}

It is known that there can be assigned to every distributive lattice with pseudocomplementation (= distributive $p$-algebra) $L$ a Boolean algebra $B(L)$ and a distributive lattice $D(L)$ with 1 (see [1] or [3]). Moreover, every congruence $\theta$ on $L$ can be represented as a congruence pair $\left(\theta_{1}, \theta_{2}\right)$ for suitable $\theta_{1} \in C(B(L))$ and $\theta_{2} \in C(D(L))$. G. Grätzer in his book [1] set a task (Problem 57): “Let $B$ be a Boolean lattice, let $D$ be a distributive lattice with unit, and let $A$ be a sublattice of $C(B) \times C(D)$. Under what conditions does there exist a distributive lattice with pseudocomplementation $L$ such that $B(L)=B, D(L)=$ $D$, and A consists of all congruence pairs of $L$ ?"

In this note we shall give an answer to this problem (Corollary 1 to Theorem 1). In fact we prove more. We shall describe the lattice of all congruence pairs of a modular $p$-algebra (Theorem 1). Theorem 2 shows that all conditions occurring in Theorem 1 are mutually independent.

Preliminaries. A universal algebra $\left\langle L ; \vee, \wedge,{ }^{*}, 0,1\right\rangle$ of type $\langle 2,2,1,0,0\rangle$ is called a modular (distributive) p-algebra iff $\langle L ; \vee, \wedge, 0,1\rangle$ is a bounded modular (distributive) lattice such that for every $a \in L$ the element $a^{*}$ is the pseudocomplement of $a$, i.e. $x \leqslant a^{*}$ iff $a \wedge x=0$. The standard results on $p$ algebras can be found in [1].

Let $L$ be a modular $p$-algebra. Define the set $B(L)=\left\{x \in L: x=x^{* *}\right\}$ of closed elements. $\left\langle B(L) ; \vee, \wedge,{ }^{*}, 0,1\right\rangle$, where $a \vee b=\left(a^{*} \wedge b^{*}\right)^{*}$, forms a Boolean algebra. Another significant subset of a modular $p$-algebra $L$ is the set of dense elements $D(L)=\left\{x \in L: x^{*}=0\right\} . D(L)$ is a filter (dual ideal) in $L$.

For an arbitrary lattice $L$, the set $F(L)$ of all filters of $L$ ordered under set inclusion is a lattice. It is known that $F(L)$ is modular (distributive) iff $L$ is modular (distributive).

For a modular $p$-algebra $L$ consider the map $\varphi(L): B(L) \rightarrow F(D(L))$ defined in the following way:

Received by the editors October 2, 1974 and, in revised form, November 3, 1974 and June 24, 1975.

AMS (MOS) subject classifications (1970). Primary 06A25, 06A30, 06A35; Secondary 06A23, 06A40.

Key words and phrases. Distributive lattice with pseudocomplementation, modular p-algebra, distributive $p$-algebra, Boolean algebra, pseudocomplement, closed element, dense element, congruence, congruence pair, modular lattice, distributive lattice. 


$$
a \varphi(L)=\left\{x \in D(L): x \geqslant a^{*}\right\}=\left[a^{*}\right) \wedge D(L), \quad a \in B(L) .
$$

By $[4$, Theorem 1], the triple $\langle B(L), D(L), \varphi(L)\rangle$ determines uniquely every modular $p$-algebra $L$.

Let $\theta$ be a congruence on a modular $p$-algebra $L$. Denote by $\theta_{B}$ and $\theta_{D}$ the restriction of $\theta$ to $B(L)$ and $D(L)$, respectively. It is easy to see that $\left(\theta_{B}, \theta_{D}\right) \in C(B(L)) \times C(D(L))$. An arbitrary pair $\left(\theta_{1}, \theta_{2}\right) \in C(B(L))$ $\times C(D(L))$ will be called a congruence pair if $a \in B(L), u \in D(L), u \geqslant a$, and $a \equiv 1 \quad\left(\theta_{1}\right)$ imply that $u \equiv 1 \quad\left(\theta_{2}\right)$.

In $[4$, Theorem 11] the following result has been proved.

Theorem A. Let $L$ be a modular p-algebra. Then every congruence $\theta$ of $L$ determines a congruence pair $\left(\theta_{B}, \theta_{D}\right)$. Conversely, every congruence pair $\left(\theta_{1}, \theta_{2}\right)$ uniquely determines a congruence $\theta$ on $L$ with $\theta_{B}=\theta_{1}$ and $\theta_{D}=\theta_{2}$ by the following rule:

$$
x \equiv y(\theta) \text { iff } \quad \text { (i) } x^{*} \equiv y^{*}\left(\theta_{1}\right) \text { and } \quad \text { (ii) } x \vee x^{*} \equiv y \vee y^{*}\left(\theta_{2}\right)
$$

In [1, Theorem 15.7] and [3, Satz 5] Theorem A was proved for a distributive p-algebra.

The main result. Let $L$ be a lattice and $H$ an ideal or filter of $L$. We denote by $\theta[H]$ the smallest congruence of $L$ such that $a \equiv b$ for all $a, b \in H$. Evidently, if $L$ is distributive, then $H$ is a congruence class of $\theta[H]$ (see [1]).

$\Delta_{L}$ and $\nabla_{L}$ denote the identity and the universal congruence on $L$, respectively.

Theorem 1. Let $\left\langle B ; \vee, \wedge,{ }^{\prime}, 0,1\right\rangle$ be a Boolean algebra, let $\langle D ; \vee, \wedge, 1\rangle$ be a modular lattice with unit, and let $A$ be a subset of $C(B) \times C(D)$. Then $A$ is the set of all congruence pairs of a modular p-algebra $L$ with $B(L)=B$ and $D(L)=$ $D$ iff the following conditions are fulfilled:

(i) $A$ is a join-complete sublattice of $C(B) \times C(D)$ containing $\left(\Delta_{B}, \Delta_{D}\right)$;

(ii) for every $a \in B$ there exists a filter $F_{a} \in F(D)$ such that $(\theta[(a]], \phi) \in A$ iff $\phi \in\left[\theta\left[F_{a}\right], \nabla_{D}\right]$

(iii) $F_{a} \wedge F_{a^{\prime}}$ is a principal filter for every $a \in B$;

(iv) the map $a \rightarrow F_{a}$ is $a\{0,1, \vee\}$-homomorphism of $B$ into $F(D)$.

Proof. Necessity. Let $A$ be the set of all congruence pairs of a modular $p$ algebra $L$ with $B(L)=B$ and $D(L)=D$. Let $\left\{\left(\psi_{i}, \phi_{i}\right): i \in I\right\} \subseteq A$ for $I \neq \varnothing$. It is easy to check that $\left(\wedge \psi_{i}, \wedge \phi_{i}\right) \in A$ and $\left(\Delta_{B}, \Delta_{D}\right) \in A$. Let $a \equiv 1 \quad\left(\vee \psi_{i}: i \in I\right)$ in the Boolean algebra $B$ and let $u \geqslant a, u \in D$. Then there exist elements $a_{1}, \ldots, a_{k} \in B$ ( $k$ finite) such that $a_{i}^{*} \equiv 1\left(\psi_{j_{i}}\right), i=1$, $\ldots, k$, and $a=a_{1}^{*} \wedge \cdots \wedge a_{k}^{*}$. Clearly, $u \geqslant a$ is equivalent to

$$
u \in\left(a_{1} \vee \cdots \vee a_{k}\right) \varphi(L) \text {. }
$$

By [4, Lemma 1], $\left(a_{1} \vee \cdots \vee a_{k}\right) \varphi(L)=a_{1} \varphi(L) \vee \cdots \vee a_{k} \varphi(L)$; therefore there exist $v_{i} \in a_{i} \varphi(L), i=1, \ldots, k$, such that $u \geqslant v_{1} \wedge \cdots \wedge v_{k}$. Evidently, $v_{i} \equiv 1\left(\phi_{j_{i}}\right), i=1, \ldots, k$. Hence, $u \equiv 1\left(\vee \phi_{i}: i \in I\right)$. Thus $\left(\vee \psi_{i}, \vee \phi_{i}\right)$ $\in A$ and (i) is proved. We prove now (ii). Let $a \in B$. Since $a \varphi(L)$ 
$=\left\{x \in D(L): x \geqslant a^{*}\right\}$, we see that $(\theta[(a]], \theta[a \varphi(L)])$ is a congruence pair of $L$, i.e. $(\theta[(a]], \theta[a \varphi(L)]) \in A$. Set $F_{a}=a \varphi(L), a \in B$. Clearly, $\phi \geqslant \theta\left[F_{a}\right]$ implies $(\theta[(a]], \phi) \in A$. Conversely, suppose $(\theta[(a]], \phi) \in A$. Evidently $a^{*}$ $\equiv 1(\theta[(a]])$. Moreover $u \in D$ and $u \geqslant a^{*}$ is equivalent to $u \in a \varphi(L)$. Hence $\Phi \geqslant \theta\left[F_{a}\right]$ and (ii) is established. (iii) follows from the fact that $F_{a}=a \varphi(L)(a$ $\in B)$ and $a \varphi(L) \wedge a^{*} \varphi(L)=\left[a \vee a^{*}\right)$. Finally, by [4, Lemma 1], the map $\varphi(L): B \rightarrow F(D)$ is a $\{0,1, \vee\}$-homomorphism, which proves (iv).

Sufficiency. First we derive from (i) and (ii) the following condition.

(ii') For every $\psi \in C(B)$ there exists a filter $F_{\psi} \in F(D)$ such that $(\psi, \phi)$ $\in A$ iff $\phi \in\left[\theta\left[F_{\psi}\right], \nabla_{D}\right]$. If $\psi=\theta[J], J$ is an ideal of $B$, then

$$
F_{\psi}=\vee\left(F_{a}: a \in J\right) .
$$

Let $\psi \in C(B)$. Then there exists an ideal $J$ of $B$ such that $\psi=\theta[J]$. Suppose $(\psi, \phi) \in A$. By (i) and (ii), we have

$$
(\psi, \phi) \wedge\left(\theta[(a]], \theta\left[F_{a}\right]\right)=\left(\theta[(a]], \theta\left[F_{a}\right] \wedge \phi\right) \in A
$$

for every $a \in J$. Hence, by (ii), $\phi \geqslant \theta\left[F_{a}\right]$, and consequently

$$
\phi \geqslant \vee\left(\theta\left[F_{a}\right]: a \in J\right) .
$$

By [2, Theorem 6],

$$
\phi \geqslant \vee\left(\theta\left[F_{a}\right]: a \in J\right)=\theta\left[\vee\left(F_{a}: a \in J\right)\right]=\theta\left[F_{\psi}\right] .
$$

Since $\left(\theta[(a]], \theta\left[F_{a}\right]\right) \in A$ for every $a \in J$, we obtain by (i) and [2, Theorem 6],

$$
\vee\left(\theta[(a]], \theta\left[F_{a}\right]\right)=\left(\theta[J], \vee \theta\left[F_{a}\right]\right)=\left(\psi, \theta\left[F_{\psi}\right]\right) \in A,
$$

and the first part of (ii') is proved. Assume $\phi \in\left[\theta\left[F_{\psi}\right], \nabla_{D}\right]$. Then by (ii), $(\theta[(a]], \phi) \in A$ for every $a \in J$. Since $\left(\psi, \theta\left[F_{\psi}\right]\right) \in A$, we get by (i),

$$
(\theta[(a]], \phi) \vee\left(\psi, \theta\left[F_{\psi}\right]\right)=(\psi, \phi) \in A,
$$

and (ii') is established. Now we show that there exists a modular $p$-algebra $L$, such that $B(L)=B$ and $D(L)=D$. If $B$ is a one-element Boolean algebra then $D$ is also a one-element lattice, by (i) and (iv). Then $A$ is the set of all congruence pairs of the one-element $p$-algebra $L$. Let $B$ be nontrivial. By (iii) and (iv), we have a map $\varphi: B \rightarrow F(D)$ with a $\varphi=F_{a}$ for every $a \in B$, which is a $\{0,1, \vee\}$-homomorphism. So, $\langle B, D, \varphi\rangle$ forms a modular triple in the sense of [4] and by [4, Theorem 4], there exists a modular $p$-algebra $L$, such that $B(L)=B, D(L)=D$ and $\varphi(L)=\varphi$. Let $A(L)$ denote the lattice of all congruence pairs of $L$. We have to prove $A(L)=A$. Suppose $(\psi, \phi) \in A, \psi$ $=\theta[J], J$ is an ideal of $B$. By (ii), (ii ') and [2, Theorem 6], we have

$$
\begin{aligned}
(\psi, \phi) & \geqslant\left(\psi, \theta\left[F_{\psi}\right]\right)=\left(\theta[J], \theta\left[\vee\left(F_{a}: a \in J\right)\right]\right) \\
& =\left(\vee \theta[(a]], \vee \theta\left[F_{a}\right]\right)=\vee\left(\theta[(a]], \theta\left[F_{a}\right]\right) \in A .
\end{aligned}
$$

By the definition of the modular $p$-algebra $L,(\theta[(a]], \theta[a \varphi])$ is a congruence pair 
of $L$ for every $a \in J$ (we know that $F_{a}=a \varphi$ ), i.e. $(\theta[(a]], \theta[a \varphi]) \in A(L)$. Since $A(L)$ is a join-complete sublattice of $C(B) \times C(D)$ (see (i)), we obtain

$$
\left(\psi, \theta\left[F_{\psi}\right]\right)=\vee((\theta[(a]], \theta[a \varphi]): a \in J) \in A(L) .
$$

By the definition of a congruence pair the last fact yields $(\psi, \phi) \in A(L)$. Thus $A \subseteq A(L)$. Analogously we can prove the converse inclusion. Actually, suppose $(\psi, \phi)$ is a congruence pair of $L$, i.e. $(\psi, \phi) \in A(L)$. Then there exists an ideal $J$ of $B$ such that $\psi=\theta[J]$. We know that $F_{a}=a \varphi=a \varphi(L)$ for every $a \in B$. Therefore $F_{\psi}=\vee(a \varphi(L): a \in J)$. Similarly as above (see the proof of (ii')) we can prove $\left(\psi, \theta\left[F_{\psi}\right]\right) \in A(L)$ in the $p$-algebra $L$ and $\theta\left[F_{\psi}\right] \leqslant \phi$ in $D(L)$. Therefore (using [2, Theorem 6])

$$
\begin{aligned}
(\psi, \phi) & \geqslant\left(\psi, \theta\left[F_{\psi}\right]\right)=\left(\theta[J], \theta\left[F_{\psi}\right]\right)=(\theta[\vee(a]], \theta[\vee a \varphi(L)]) \\
& =(\vee \theta[(a]], \vee \theta[a \varphi(L)])=\vee((\theta[(a]], \theta[a \varphi(L)]): a \in J) \in A,
\end{aligned}
$$

because $(\theta[(a]], \theta[a \varphi(L)]) \in A$ for any $a \in J$ and $A$ is a join-complete sublattice of $C(B) \times C(D)$, by (i). The last fact infers $(\psi, \phi) \in A$, by (ii'). Hence $A(L) \subseteq A$. Concluding we obtain $A(L)=A$, as claimed, and the proof is finished.

The following statement yields a solution to the above mentioned Grätzer's problem [1, Problem 57].

Corollary 1. Let $B$ be a Boolean algebra, let $D$ be a distributive lattice with 1 and let $A$ be a subset of $C(B) \times C(D)$. Then $A$ is the set of all congruence pairs of a distributive p-algebra $L$ with $B(L)=B$ and $D(L)=D$ iff conditions (i)-(iii) from Theorem 1 together with the condition

(iv') $\left(\nabla_{B}, \phi\right) \in A$ implies $\phi=\nabla_{D}$ are satisfied.

Proof. The necessity follows from Theorem 1. As for the sufficiency, we prove that (i)-(iii) and (iv') imply (iv). Examine the map $a \rightarrow F_{a}$ of $B$ into $F(D)$. By (i), $F_{0}=\left[1\right.$ ), and $F_{1}=D$, by (iv'). Suppose, $a, b \in B$. By (ii) we have

$$
\left(\theta[(a]], \theta\left[F_{a}\right]\right),\left(\theta[(b]], \theta\left[F_{b}\right]\right),\left(\theta[(a \vee b]], \theta\left[F_{a \vee b}\right]\right) \in A \text {. }
$$

Using (i) and (ii) it is easy to prove $\theta\left[F_{a}\right], \theta\left[F_{b}\right] \leqslant \theta\left[F_{a \vee b}\right]$, and hence $F_{a}, F_{b} \subseteq F_{a \vee b}$. (We recall that $F_{c}, c \in B$, is a congruence class of $\theta\left[F_{c}\right]$.) Therefore, $F_{a} \vee F_{b} \subseteq F_{a \vee b}$. On the other hand,

$$
\left(\theta[(a]], \theta\left[F_{a}\right]\right) \vee\left(\theta[(b]], \theta\left[F_{b}\right]\right)=\left(\theta[(a \vee b]], \theta\left[F_{a} \vee F_{b}\right]\right) \in A,
$$

by (i) and [2, Theorem 6]. Therefore, $\theta\left[F_{a} \vee F_{b}\right] \geqslant \theta\left[F_{a \vee b}\right]$, by (ii). This implies $F_{a \vee b} \subseteq F_{a} \vee F_{b}$. Concluding we have $F_{a \vee b}=F_{a} \vee F_{b}$. So, we have proved (iv), i.e. the map $a \rightarrow F_{a}$ is a $\{0,1, \vee\}$-homomorphism of $B$ into $F(D)$. The rest of the proof follows from Theorem 1.

Before stating the following corollary we need two more concepts. A modular (distributive) $p$-algebra is said to be a modular S-algebra (Stone algebra) if it satisfies the identity $x^{*} \vee x^{* *}=1$. 
Corollary 2. Let $B$ be a Boolean algebra, let $D$ be a modular (distributive) lattice with 1 and let $A$ be a subset of $C(B) \times C(D)$. Then $A$ is the set of all congruence pairs of a modular $S$-algebra (Stone algebra) $L$ with $B(L)=B$ and $D(L)=D$ iff conditions (i), (ii), (iv) from Theorem 1 together with the condition (iii') $F_{a} \wedge F_{a^{\prime}}=[1)$ for every $a \in B$

((i), (ii), (iii') and (iv')) are satisfied.

The proof follows from Theorem 1, Corollary 1 and [4].

REMARK. Going through the proofs of Theorem 1 and Corollary 1 we see that in case $D$ is distributive, the corresponding distributive $p$-algebra $L$, with $B(L)=B, D(L)=D$ and $A$ is the set of all congruence pairs of $L$, is uniquely determined. The following example shows that this does not hold in general.

EXAMPLE. In Figure 1 are pictured two distinct modular $p$-algebras $L$ and $L_{1}$ with $B(L)=B\left(L_{1}\right), D(L)=D\left(L_{1}\right)$ and having the same lattice of all congruence pairs.
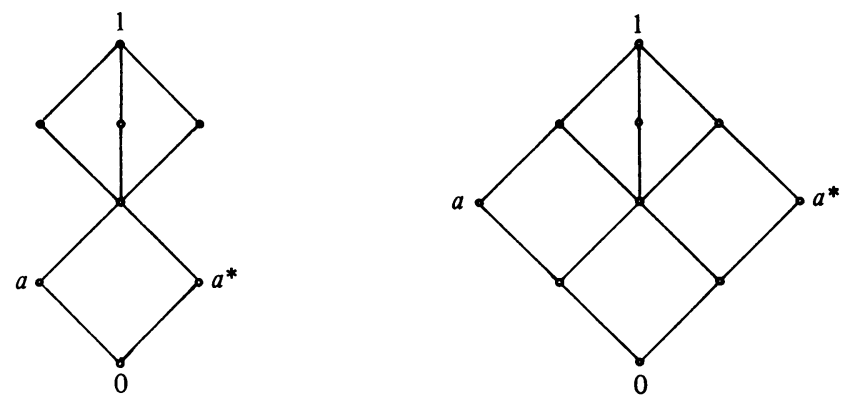

Figure 1

THEOREM 2. The following systems of conditions are independent:

(1) (i)-(iv) from Theorem 1;

(2) (i)-(iii) and (iv') from Corollary 1 to Theorem 1;

(3) (i), (ii), (iv) and (iii') from Corollary 2 to Theorem 1;

(4) (i), (ii), (iii') and (iv') from Corollary 2 to Theorem 1.

Proof. (i) Let $B$ be an infinite Boolean algebra. Let $D=B$. Define the set $A \subseteq C(B) \times C(D)$ as follows: $(\psi, \phi) \in A$ iff

(a) $\operatorname{Ker} \psi=\{x \in B: x \equiv 0 \quad(\psi)\}$ is not principal then $\phi=\nabla_{D}$, or

(b) $J=\operatorname{Ker} \psi$ is principal then $\phi \in\left[\theta\left[J^{\prime}\right], \nabla_{D}\right]$, where $J^{\prime}=\left\{x \in B: x^{\prime}\right.$ $\in J\}$. It is easy to check that conditions (ii)-(iv), (iii') and (iv') are satisfied. Now we show that condition (i) fails to hold, namely, that $A$ is not a sublattice of $C(B) \times C(D)$. The Boolean algebra $B$ contains an ideal $J$ which is not principal. Let $0 \neq a \neq 1$ be an element of $B$. Then $(a] \wedge J$ or $\left(a^{\prime}\right] \wedge J$ is not principal. Suppose $(a] \wedge J$ is such an ideal. Evidently $\left(\theta[(a]], \theta\left[\left[a^{\prime}\right)\right]\right) \in A$ and $\left(\theta[J], \nabla_{D}\right) \in A$. But

$$
\left(\theta[(a]], \theta\left[\left[a^{\prime}\right)\right]\right) \wedge\left(\theta[J], \nabla_{D}\right)=\left(\theta[(a] \wedge J], \theta\left[\left[a^{\prime}\right)\right]\right) \notin A,
$$

since $\theta\left[\left[a^{\prime}\right)\right] \neq \nabla_{D}$ and $(a] \wedge J$ is not principal.

(ii) Let $B$ be the four-element Boolean algebra $\left\{0, a, a^{\prime}, 1\right\}$ and let $B=D$. Define the set $A \subseteq C(B) \times C(D)$ as follows: 


$$
A=\left\{\left(\Delta_{B}, \Delta_{D}\right),\left(\theta[(a]], \theta\left[\left[a^{\prime}\right)\right]\right),\left(\theta\left[\left(a^{\prime}\right]\right], \theta[[a)]\right),\left(\nabla_{B}, \nabla_{D}\right)\right\} .
$$

One can easily verify that (ii) does not hold and that (i), (iii), (iii'), (iv) and (iv') are true.

(iii) Let $B$ be the four-element Boolean algebra $\left\{0, a, a^{\prime}, 1\right\}$, and $D$ an infinite Boolean algebra. It is not difficult to show that $D$ contains two distinct filters $F_{a}$ and $F_{a^{\prime}}$ such that $F_{a} \vee F_{a^{\prime}}=D$ and $F_{a} \wedge F_{a^{\prime}}$ is not principal. Define the set $A \subseteq C(B) \times C(D)$ as follows. $(\psi, \phi) \in A$ iff

(a) $\psi=\Delta_{B}, \phi \in C(D)$

(b) $\psi=\theta[(a]], \phi \in\left[\theta\left[F_{a}\right], \nabla_{D}\right]$;

(c) $\psi=\theta\left[\left(a^{\prime}\right]\right], \phi \in\left[\theta\left[F_{a^{\prime}}\right], \nabla_{D}\right]$;

(d) $\psi=\nabla_{B}, \phi=\nabla_{D}$.

It is easy to check that conditions (i), (ii), (iv) and (iv') hold but (iii) and (iii') do not.

(iv) Let $B$ be the four-element Boolean algebra $\left\{0, a, a^{\prime}, 1\right\}$ and $D$ the threeelement chain $e_{1}<e_{2}<e_{3}$. Define the set $A \subseteq C(B) \times C(D)$ as follows: $(\psi, \phi) \in A$ iff

(a) $\psi \in\left\{\Delta_{B}, \theta[(a]]\right\}, \phi \in C(D)$;

(b) $\psi \in\left\{\theta\left[\left(a^{\prime}\right]\right], \nabla_{B}\right\}, \phi \in\left[\theta\left[\left[e_{2}\right)\right], \nabla_{D}\right]$.

Conditions (i)-(iii) and (iii') are satisfied, but (iv) and (iv') are not.

\section{REFERENCES}

1. G. Grätzer, Lattice theory. First concepts and distributive lattices, Freeman, San Francisco, Calif., 1971.

2. G. Grätzer and E. T. Schmidt, Ideals and congruence relations in lattices, Acta Math. Acad. Sci. Hungar. 9 (1958), 137-175. MR 20 \#6990.

3. T. Katriňák, Über eine Konstruktion der distributiven pseudokomplementären Verbände, Math. Nachr. 53 (1972), 85-99. MR 47 \# 4882.

4. T. Katrińák and P. Mederly, Construction of modular p-algebras, Algebra Universalis 4 (1974), 301-315.

Katedra Algebry a Té́rie Čísel PFUK, 81631 Bratislava 16-Mlynská Dolina,CzechoSLOVAKIA 